\title{
Indirect arterial blood pressure measurement in healthy anesthetized cats using a device that combines oscillometry with photoplethysmography
}

\author{
Yasuhiro HEISHIMA ${ }^{1,2)}$, Yasutomo HORI ${ }^{2}$ *, Seishiro CHIKAZAWA ${ }^{2)}$, Kazutaka KANAI ${ }^{2)}$, Fumio HOSHI $^{2)}$ and \\ Naoyuki ITOH ${ }^{2)}$ \\ ${ }^{1)}$ Heisei Animal Hospital, 2-1-1 Futagocho, Kasugai, Aichi 486-0955, Japan \\ 2) Laboratory of Small Animal Internal Medicine II, School of Veterinary Medicine, Kitasato University, 23-35-1 Higashi, Towada, \\ Aomori 034-8628, Japan
}

(Received 27 October 2015/Accepted 7 March 2016/Published online in J-STAGE 18 March 2016)

ABSTRACT. We investigated the basic characteristics of indirect arterial blood pressure (ABP) measurement using a device that combines oscillometry and photoplethysmography in cats. Dobutamine was infused intravenously in four anesthetized cats. Direct ABP was measured by a catheter. Indirect ABP was measured from the left forelimb. Dobutamine significantly elevated both systolic arterial pressure (SAP) and mean arterial pressure (MAP) in a dose-dependent manner. The indirect SAP, MAP and diastolic arterial pressure (DAP) values were closely correlated with the direct ABP values $(r=0.88,0.89$ and 0.83 , respectively). The mean bias for SAP, MAP and DAP was $3.4,0.2$ and $-2.4 \mathrm{mmHg}$, respectively. The indirect ABP measured by this device may be used to reliably monitor ABP changes in anesthetized cats. KEY WORDS: blood pressure, feline, oscillometry, photoplethysmography, systemic hypertension

doi: 10.1292/jvms.15-0612; J. Vet. Med. Sci. 78(7): 1179-1182, 2016

The measurement of arterial blood pressure (ABP) is essential for monitoring anesthetized cats and for the diagnosis and management of systemic hypertension in cats. Direct $\mathrm{ABP}$ measurement is considered the gold standard of ABP measurement; however, cats require general anesthesia to obtain an accurate reading. In contrast, indirect $\mathrm{ABP}$ measurement does not require general anesthesia, and it is available for repeated monitoring. For this reason, several methods of indirect ABP measurement are utilized, including Doppler, oscillometric and photoplethysmographic techniques. Of these, oscillometric ABP measurement is the most often used in cats and dogs. Some clinical studies have shown that indirect $\mathrm{ABP}$ measurement using oscillometry may provide diagnostic information related to systemic hypertension in cats $[4,6,14]$. Although agreement and accuracy vary between the approaches $[1,2,7,11,12]$, indirect systolic arterial pressure (SAP) measurements that used an oscillometric technique were highly correlated with direct SAP in anesthetized cats $[2,7]$.

Photoplethysmography is a non-invasive technique that measures blood volume changes from the peripheral circulation. When the heart pumps blood to the periphery, the pressure pulse distends the arteries and arterioles in the subcutaneous tissue. The change in blood volume caused by the pressure pulse is detected by illuminating the skin in these regions with low-level infrared light. Photoplethysmography depends on the fact that blood absorbs infrared light much

Correspondence to: Hori, Y., School of Veterinary Medicine, Rakuno Gakuen University, 582 Midoricho, Bunkyodai, Ebetsu, Hokkaido 069-8501, Japan. e-mail: hori@vmas.kitasato-u.ac.jp (C)2016 The Japanese Society of Veterinary Science

This is an open-access article distributed under the terms of the Creative Commons Attribution Non-Commercial No Derivatives (by-nc-nd) License $<$ http://creativecommons.org/licenses/by-nc-nd/4.0/>. more strongly than the surrounding tissues. It has been used widely in many medical applications, such as pulse oximetry. A new device (BP100D, FUKUDA ME, Tokyo, Japan) that combines oscillometry and photoplethysmography has become available in veterinary practice. However, no study has assessed the indirect $\mathrm{ABP}$ obtained by this device in cats.

The American College of Veterinary Internal Medicine (ACVIM) has published guidelines for the validation of new devices for ABP measurement to detect hypertension in companion animals [5]. The ACVIM guidelines require that the correlation between paired measures for ABPs taken separately is greater than 0.9 . Good agreement between indirect and direct $\mathrm{ABP}$ is defined as a mean difference of less than $10 \mathrm{mmHg}$ and a standard deviation (SD) within $15 \mathrm{mmHg}$ [5]. Finally, $50 \%$ and $80 \%$ of all indirect ABP measurements should be within 10 and $20 \mathrm{mmHg}$ of the reference method, respectively [5]. Although the ACVIM guidelines are targeted at detecting hypertension in conscious animals, the ACVIM consensus statement on ABP measurements was used to validate the utility of BP100D in anesthetized cats. The purpose of this present study was to investigate whether a BP100D-derived indirect ABP measurement reflected the changes in direct ABP measurements in anesthetized cats.

The present study was conducted in accordance with the Guidelines for Institutional Laboratory Animal Care and Use promulgated by the School of Veterinary Medicine and Animal Science at Kitasato University. Four adult mixedbreed cats (three males and one female), ages 1-3 (mean 1.5) years and weighing 3.0-6.0 (mean 4.1) kg, were used. All cats were healthy on physical examination, blood chemistry analysis and echocardiography.

The cats were sedated by administering butorphanol $(0.2 \mathrm{mg} / \mathrm{kg}$, intravenously [iv], Meiji Seika Pharma, Tokyo, Japan), diazepam $(0.5 \mathrm{mg} / \mathrm{kg}$, iv, Takeda Pharmaceutical, Osaka, Japan $)$ and atropine $(0.025 \mathrm{mg} / \mathrm{kg}$, subcutane- 
Table 1. Changes in hemodynamic measurements during dobutamine administration

\begin{tabular}{llrcc}
\hline & \multirow{2}{*}{ Baseline } & \multicolumn{2}{c}{ Dobutamine } \\
\cline { 3 - 4 } & & $5 \mu \mathrm{g} / \mathrm{kg} / \mathrm{min}$ & $10 \mu \mathrm{g} / \mathrm{kg} / \mathrm{min}$ \\
\hline Heart rate (bpm) & Direct & $152 \pm 28$ & $193 \pm 38$ & $\left.205 \pm 43^{\mathrm{a}}\right)$ \\
& Indirect & $151 \pm 18$ & $187 \pm 35^{\mathrm{a})}$ & $\left.214 \pm 37^{\mathrm{b}}\right)$ \\
$\mathrm{SAP}(\mathrm{mmHg})$ & Direct & $83 \pm 14$ & $102 \pm 17^{\mathrm{a})}$ & $106 \pm 22^{\mathrm{b})}$ \\
& Indirect & $83 \pm 14$ & $\left.102 \pm 13^{\mathrm{a}}\right)$ & $99 \pm 15^{\mathrm{a})}$ \\
$\mathrm{MAP}(\mathrm{mmHg})$ & Direct & $61 \pm 16$ & $\left.73 \pm 17^{\mathrm{a}}\right)$ & $74 \pm 16^{\mathrm{a}}$ \\
& Indirect & $59 \pm 14$ & $76 \pm 15^{\mathrm{a})}$ & $74 \pm 19^{\mathrm{a})}$ \\
$\mathrm{DAP}(\mathrm{mmHg})$ & Direct & $48 \pm 16$ & $55 \pm 15$ & $54 \pm 14$ \\
& Indirect & $45 \pm 14$ & $61 \pm 16$ & $59 \pm 20$ \\
\hline
\end{tabular}

Data are means \pm SDs. SAP: systolic arterial pressure, MAP: mean arterial pressure, DAP: diastolic arterial pressure. a) $P<0.05$ vs. baseline, b) $P<0.01$ vs. baseline.

ously, Mitsubishi Tanabe Pharma, Osaka, Japan). After a 15-30 min stabilization period, anesthesia was induced by administration of propofol $(6 \mathrm{mg} / \mathrm{kg}$, iv, Inervet, Tokyo, Japan), and each cat was intubated. Anesthesia was maintained using a mixture of isoflurane (2.0-3.0\%, Inervet, Tokyo, Japan) in oxygen. After $30 \mathrm{~min}$ of anesthesia, we confirmed loss of the eyelid and laryngeal reflexes and stabilization of the heart rate, respiratory rate and ABP. The respiratory rate was maintained at 20 breaths/min using an artificial ventilator. End-tidal $\mathrm{CO}_{2}$ was maintained between 35 and $45 \mathrm{mmHg}$, arterial oxygen saturation $\left(\mathrm{SPO}_{2}\right)$ was maintained between 95 and $100 \%$, and heart rate and direct $\mathrm{ABP}$ were monitored using the multifunction monitor (COLIN BP-608, COLIN, Tokyo, Japan). Dobutamine (Dobutrex, Shionogi \& Co., Osaka, Japan) was infused at 5.0 and $10 \mu \mathrm{g} / \mathrm{kg} / \mathrm{min}$ for 5-10 min via the right cephalic vein [8]. Measurements were performed at baseline and after each dose of dobutamine.

The SAP, mean arterial pressure (MAP) and diastolic arterial pressure (DAP) were measured by both direct and indirect methods. The anesthetized cat was positioned in right lateral recumbency. For direct ABP measurements, a 3 Fr fluid-filled catheter was inserted into the right femoral artery. The catheter was connected to a disposable pressure transducer (TruWave Disposable Pressure Transducer, Nihon Kohden, Tokyo, Japan) through an extension tube (TOP blood pressure monitoring tube, TOP Co., Tokyo, Japan). The transducer was placed at the level of the heart and connected to a continuous multifunction monitor (COLIN BP-608). Zero calibration was performed using the multifunction monitor (atmospheric pressure $=0 \mathrm{mmHg}$ ). The accuracy of the direct ABP was judged from the stability and consistency of the waveform.

Indirect ABPs were measured by a device that combines oscillometry and photoplethysmography. The appropriate cuff size (40-60\% of the limb circumference) was chosen according to the manufacturer's instructions, and a No. 3 (cuff width $4.0 \mathrm{~cm}$ and cuff limb circumference 6-11 cm) was used. The cuff was placed onto the left forelimb distal to the elbow. A total of four readings were taken under each condition. The corresponding direct ABP readings were recorded simultaneously. Intra-cat coefficients of variabilities were $6.9 \%$ at a mean SAP of $82.6 \mathrm{mmHg}$ and $7.2 \%$ at a mean SAP of $98.9 \mathrm{mmHg}$.

Statistical analyses were performed using statistical software (Stat Mate III, Avice, Inc., Tokyo, Japan). Data are given as the means \pm SD. The effects of different dobutamine doses were evaluated using one-factor repeated measures analysis of variance. If a significant difference was found between the baseline and dobutamine dosage, a post hoc multiple comparison was conducted using Tukey's test. Correlation coefficients ( $r$ values) and regression equations were calculated to compare the two methods of ABP measurement. A $P$-value $<0.05$ was considered to indicate significance.

Bland-Altman analysis for multiple observations per individual was performed using statistical software (MedCalc version 15.2.2, MedCalc Software bvba, Ostend, Belgium) [3]. Differences in ABP between the two methods were calculated as direct ABP -indirect ABP. The bias between the two measures was assessed from the mean difference of the two methods, and 95\% limits of agreement (LOA) were calculated as bias $\pm(1.96 \times \mathrm{SD}$ of the differences $)[3,9,11]$. A positive or negative bias would reflect an underestimation or overestimation of the ABP values by indirect ABP measurement in relation to direct ABP measurement. The agreement was assessed from the absolute mean values of the differences and the SD of the two methods.

Hemodynamic reactions caused by dobutamine were dose-dependent (Table 1). The heart rate increased significantly from baseline. Also, the SAP and MAP increased significantly (both direct and indirect measurements). Significant correlations were evident between the direct and indirect SAP measurements $(r=0.88 ; P<0.001)$, direct and indirect MAP measurements $(r=0.89 ; P<0.001)$, and direct and indirect DAP measurements $(r=0.83 ; P<0.001)$ (Fig. 1).

When the SAP values were compared, the indirect SAP values were lower than the direct values, and the bias was $3.4 \mathrm{mmHg}$ (95\% LOA; -15.9 to $22.8 \mathrm{mmHg}$, Fig. 2A). The agreement for SAP was $8.0 \pm 6.5 \mathrm{mmHg}$. The overall percentages of indirect SAP readings within 10 and $20 \mathrm{mmHg}$ of the direct SAP readings were $71.1 \%$ and $91.1 \%$, respectively. When the MAP values were compared, the indirect 

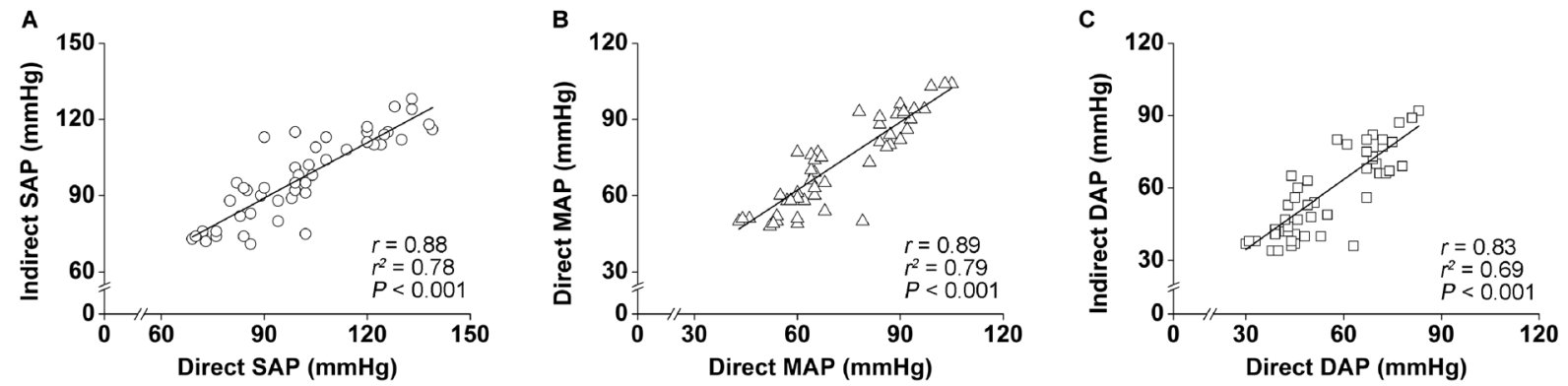

Fig. 1. Regression analysis of arterial blood pressure (measured directly and indirectly). A significant correlation was evident between the direct and indirect SAP values (A), between the direct and the indirect MAP values (B), and between the direct and the indirect DAP values (C). Indirect BP readings were made simultaneously with each direct BP reading during a study. And, a total of 48 readings were obtained. SAP: systolic arterial pressure, MAP: mean arterial pressure, DAP: diastolic arterial pressure.

A

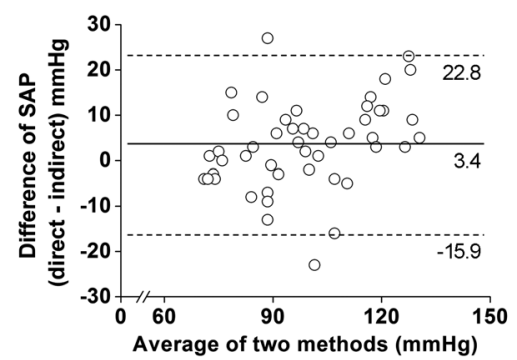

B

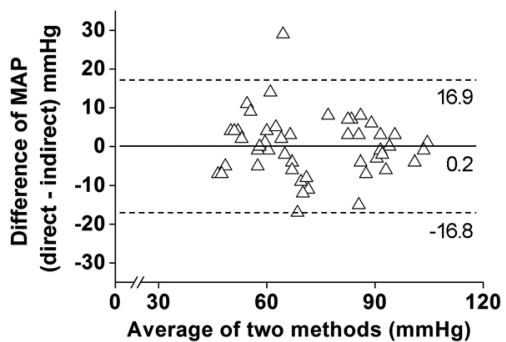

C

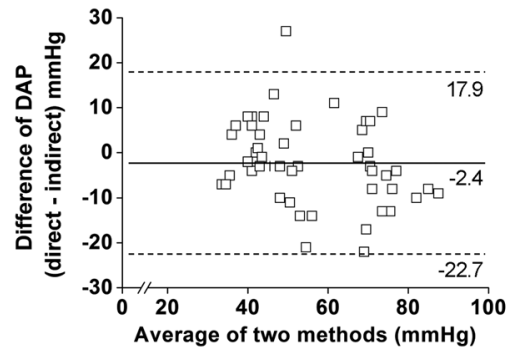

Fig. 2. Bland-Altman plot of the direct and indirect measurements of arterial blood pressure. Indirect BP readings were made simultaneously with each direct BP reading during a study. And, a total of 48 readings were obtained. The horizontal line represents the mean, and the dashed lines above and below the mean are the $+1.96 \times \mathrm{SD}$ and $-1.96 \times \mathrm{SD}$ levels, respectively. Indirect SAP measurements were lower than the direct values (A). The indirect MAP values were slightly greater than the direct values (B). The indirect DAP values were greater than the direct DAP values (C). SAP: systolic arterial pressure, MAP: mean arterial pressure, DAP: diastolic arterial pressure.

MAP values were slightly greater than the direct values, and the bias was $0.2 \mathrm{mmHg}(95 \% \mathrm{LOA} ;-16.8$ to $16.9 \mathrm{mmHg}$, Fig. 2B). The agreement for MAP was $7.9 \pm 6.2 \mathrm{mmHg}$. The overall percentages of indirect MAP readings within 10 and $20 \mathrm{mmHg}$ of the direct MAP readings were $84.4 \%$ and $97.8 \%$, respectively. When the DAP values were compared, the indirect DAP measurements predicted the direct DAP measurements with a bias of $-2.4 \mathrm{mmHg}(95 \% \mathrm{LOA} ;-22.7$ to $17.9 \mathrm{mmHg}$, Fig. 2C). The agreement for DAP was $7.9 \pm$ $6.2 \mathrm{mmHg}$. The overall percentages of indirect DAP readings within 10 and $20 \mathrm{mmHg}$ of the direct DAP readings were $75.6 \%$ and $93.3 \%$, respectively.

Dobutamine is known to stimulate beta1-adrenergic receptors, which exert inotropic and chronotropic effects. Previous studies reported that intravenous administration of dobutamine dose-dependently increased SAP at a rate of $5-10 \mu \mathrm{g} / \mathrm{kg} / \mathrm{min}$ in cats and dogs $[8,10]$. Thus, we chose to administer dobutamine at a rate of $5-10 \mu \mathrm{g} / \mathrm{kg} / \mathrm{min}$ to regulate SAP. Our study shows that the intravenous administration of dobutamine increased direct SAP in anesthetized cats, which is consistent with findings from previous studies $[8,10]$. Also, indirect SAP and MAP measurements were significantly elevated by dobutamine in a dose-dependent manner.
Previous studies in cats reported that oscillometric SAP was significantly correlated with direct SAP, but the correlation coefficients differ between reports [2, 7]. Recently, high-definition oscillometry was developed and evaluated in dogs and cats $[9,12,13]$, and indirect SAP and DAP values obtained with this method were highly correlated with direct measures in non-anesthetized cats $(r=0.92$ and 0.81 , respectively) [9]. In the present study, the indirect SAP, MAP and DAP values were closely correlated with the direct values. These results suggest that the measurement of indirect $A B P$ using the $\mathrm{BP} 100 \mathrm{D}$ reflects $\mathrm{ABP}$ changes in anesthetized cats, but the measurements did not reach ACVIM requirements.

Although bias is important when validating indirect $\mathrm{ABP}$ measurement techniques, oscillometric devices have been reported to have large biases in cats $[1,2,7]$. In the 1990s, an oscillometric device exhibited a bias of $-15.9 \mathrm{mmHg}$ in cats [7]. Another study investigated the bias between direct and indirect SAP readings obtained with three oscillometric devices in cats; the bias was reported to be $-10.3,-13.4$ and $-14.9 \mathrm{mmHg}$, respectively [1]. In contrast, high-definition oscillometry was associated with an SAP bias of only $-2.2 \mathrm{mmHg}$ in cats [9]. These bias differences may be due to the inaccuracy of the device and the algorithm used, as well as their positioning and the different anesthetic proto- 
cols used. In the present study, the BP100D biases for SAP, MAP and DAP were 3.4, 0.2 and $-2.4 \mathrm{mmHg}$, respectively. These results indicate that the novel device that combines oscillometry and photoplethysmography has small biases for ABP monitoring in anesthetized cats.

In addition to bias, agreement and accuracy are important in validating indirect ABP measurement techniques. In cats, the oscillometric technique has been reported to have poor accuracy compared with direct ABP measurement. The mean overall percentages of oscillometric readings within 10 and $20 \mathrm{mmHg}$ of the direct ABP readings were $30 \%$ and $60 \%$, respectively [2]. In contrast, a recent study reported that the number of SAP readings within 10 and $20 \mathrm{mmHg}$ between the two methods was $88 \%$ and $96 \%$, respectively [9]. These differences may be caused by device differences between oscillometry and high-definition oscillometry. Our study shows that the agreement (absolute mean difference and SD) for SAP, MAP and DAP was within 10 and $15 \mathrm{mmHg}$, respectively. The overall percentage of indirect $\mathrm{ABP}$ readings within $10 \mathrm{mmHg}$ of the direct $\mathrm{ABP}$ readings was $>70 \%$, while the overall percentage of indirect $\mathrm{ABP}$ readings within $20 \mathrm{mmHg}$ of the direct $\mathrm{ABPs}$ readings was $>90 \%$. These results indicate that this device has good agreement and accuracy for ABP monitoring in anesthetized cats.

Because our study was limited by the small sample size and we performed repeated measurements in anesthetized cats, our data are not applicable to conscious cats. Furthermore, some studies have reported wide variability for oscillometric ABP monitors in cats. For example, the correlation coefficients were 0.71 for a low SAP $(<110 \mathrm{mmHg}), 0.78$ for a normal SAP $(110-150 \mathrm{mmHg})$ and 0.87 for a high SAP $(>150 \mathrm{mmHg}$ ) [9]. In addition, the biases between the two methods for low, normal and high SAP values were -10.8 , -3.4 and $2.9 \mathrm{mmHg}$, respectively [9]. Thus, additional studies will be required to investigate the agreement and precision of this device for a wide range of ABP measurements.

The indirect ABPs measured by a novel device that combines oscillometry and photoplethysmography were closely correlated with the values determined directly. The agreement and accuracy met the validation criteria in anesthetized cats; however, large scale clinical studies will be required to validate the clinical utility of this device in cats.

\section{REFERENCES}

1. Acierno, M. J., Seaton, D., Mitchell, M. A. and da Cunha, A. 2010. Agreement between directly measured blood pressure and pressures obtained with three veterinary-specific oscillometric units in cats. J. Am. Vet. Med. Assoc. 237: 402-406. [Medline] [CrossRef]

2. Binns, S. H., Sisson, D. D., Buoscio, D. A. and Schaeffer, D. J.
1995. Doppler ultrasonographic, oscillometric sphygmomanometric, and photoplethysmographic techniques for noninvasive blood pressure measurement in anesthetized cats. J. Vet. Intern. Med. 9: 405-414. [Medline] [CrossRef]

3. Bland, J. M. and Altman, D. G. 2007. Agreement between methods of measurement with multiple observations per individual. J. Biopharm. Stat. 17: 571-582. [Medline] [CrossRef]

4. Bodey, A. R. and Sansom, J. 1998. Epidemiological study of blood pressure in domestic cats. J. Small Anim. Pract. 39: 567-573. [Medline] [CrossRef]

5. Brown, S., Atkins, C., Bagley, R., Carr, A., Cowgill, L., Davidson, M., Egner, B., Elliott, J., Henik, R., Labato, M., Littman, M., Polzin, D., Ross, L., Snyder, P., Stepien R., American College of Veterinary Internal Medicine 2007. Guidelines for the identification, evaluation, and management of systemic hypertension in dogs and cats. J. Vet. Intern. Med. 21: 542-558. [Medline] [CrossRef]

6. Carter, J. M., Irving, A. C., Bridges, J. P. and Jones, B. R. 2014 The prevalence of ocular lesions associated with hypertension in a population of geriatric cats in Auckland, New Zealand. N. Z. Vet. J. 62: 21-29. [Medline] [CrossRef]

7. Caulkett, N. A., Cantwell, S. L. and Houston, D. M. 1998. A comparison of indirect blood pressure monitoring techniques in the anesthetized cat. Vet. Surg. 27: 370-377. [Medline] [CrossRef]

8. Hori, Y., Uechi, M., Indou, A., Yamano, S., Ebisawa, T., Teshima, K. and Asano, K. 2007. Changes in the myocardial performance index during dobutamine administration in anesthetized cats. Am. J. Vet. Res. 68: 385-388. [Medline] [CrossRef]

9. Martel, E., Egner, B., Brown, S. A., King, J. N., Laveissiere, A., Champeroux, P. and Richard, S. 2013. Comparison of highdefinition oscillometry - a non-invasive technology for arterial blood pressure measurement - with a direct invasive method using radio-telemetry in awake healthy cats. J. Feline Med. Surg. 15: 1104-1113. [Medline] [CrossRef]

10. McEntee, K., Amory, H., Clercx, C., Soyeur, D., Michaux, C., Vanhaeverbeek, O., Jacqmot, O. and Henroteaux, M. 1998. Physiologic response to dobutamine infusion during cardiac stress testing of dogs. Am. J. Vet. Res. 59: 1160-1165. [Medline]

11. Pedersen, K. M., Butler, M. A., Ersbøll, A. K. and Pedersen, H. D. 2002. Evaluation of an oscillometric blood pressure monitor for use in anesthetized cats. J. Am. Vet. Med. Assoc. 221: 646-650. [Medline] [CrossRef]

12. Petric, A. D., Petra, Z., Jerneja, S. and Alenka, S. 2010. Comparison of high definition oscillometric and Doppler ultrasonic devices for measuring blood pressure in anaesthetised cats. $J$. Feline Med. Surg. 12: 731-737. [Medline] [CrossRef]

13. Rysnik, M. K., Cripps, P. and Iff, I. 2013. A clinical comparison between a non-invasive blood pressure monitor using high definition oscillometry (Memodiagnostic MD 15/90 Pro) and invasive arterial blood pressure measurement in anaesthetized dogs. Vet. Anaesth. Analg. 40: 503-511. [Medline]

14. Sansom, J., Rogers, K. and Wood, J. L. 2004. Blood pressure assessment in healthy cats and cats with hypertensive retinopathy. Am. J. Vet. Res. 65: 245-252. [Medline] [CrossRef] 